\title{
Procedimiento para la gestión por procesos: métodos y herramientas de apoyo
}

\author{
Procedure for process management: methods and support tools \\ Alberto Medina León ${ }^{1 *} \quad$ Dianelys Nogueira Rivera ${ }^{1}$ \\ Arialys Hernández-Nariño ${ }^{2}$ Raúl Comas Rodríguez ${ }^{3}$ \\ Recibido 14 de marzo de 2017, aceptado 25 de junio de 2018 \\ Received: March 14, 2017 Accepted: June 25, 2018
}

\begin{abstract}
RESUMEN
La investigación propone un procedimiento para la mejora de procesos que centra su objetivo en lograr el enfoque al cliente, el alineamiento estratégico y la mejora continua. La propuesta es el resultado del estudio y análisis estadístico de más de 80 procedimientos de mejora encontrados en la literatura, su aplicación parcial por más de 10 años en, al menos, 40 empresas de manufactura y servicio y la inserción de un conjunto de herramientas estadísticas y criterios de decisión que ayudan a su fundamentación científica, a la vez que a la reducción del tiempo de su aplicación. Por otra parte, se logra una exhaustiva representación de los procesos que contribuye a la implementación e integración de los sistemas de gestión asociados a las Normas ISO.
\end{abstract}

Palabras clave: Procedimiento de mejora, mejora de procesos, alineamiento.

\begin{abstract}
The research proposes a procedure for process improvement that focuses its goal on achieving customer focus, strategic alignment and continuous improvement. The proposal is the result of the study and statistical analysis of more than 80 improvement procedures found in the literature, its partial application for more than 10 years in at least 40 manufacturing and service companies and the insertion of a set of statistical tools and criteria of decision that help to its scientific foundation, as well as to the reduction of the time of its application. On the other hand, the exhaustive representation of the processes that are achieved with its application contributes to the implementation and integration of the management systems associated with ISO standards.
\end{abstract}

Keywords: Improvement procedure, process improvement, alignment.

\section{INTRODUCCIÓN}

La eliminación de los defectos, la mejora y la reducción del tiempo para entregar productos y servicios, son objetivos esenciales y comunes de casi todas las organizaciones. Para lograr estos objetivos se hace necesario entender primero, y después cambiar, los procesos donde ocurren las

1 Departamento de Industrial. Universidad de Matanzas. Calle 184 No 15303 e/ 153 y 155, Reparto Iglesias, Matanzas, Cuba. CP: 40100.E-mail: alberto.medina@umcc.cu; amedinaleon@gmail.com; dianelys.nogueira@umcc.cu; nelydaylinyuly@gmail.com

2 Departamento de Ciencia y Técnica. Universidad de Ciencias Médicas “Dr. Juan Guiteras Gener”. Laborde final No 6305 e/ San Isidro e Isabel $1^{\text {ra }}$. Reparto Versalles. Matanzas. Cuba, CP: 10400.

E-mail: arialys.hernandez@gmail.com, investigaciones.mtz@infomed.sld.cu

3 Universidad Regional Autónoma de los Andes. Conjunto Oriental. Módulo 3 apto 302. Ciudad de Ambato - Tugurahua, Ecuador. Email: raulcomasrodriguez@gmail.com

* Autor de correspondencia 
ineficacias, defectos, baja satisfacción o el bajo ritmo de producción [1].

Tradicionalmente ha existido el enfoque funcional o por departamentos que contrasta con el enfoque por procesos referida por autores como como Zaratiegui (1999) [2] y abordado en trabajos de más actualidad como Hernández Nariño (2013) para el sector hospitalario [3]; Arango Serna (2015) [4] y Flores Santander (2017) [5] en estudios de mejora de procesos y Castro Silva (2017) [6] asociado a la implementación de Normas ISO \{ Amozarrain, 1999 \#3;Amozarrain, 1999 $\# 3$ \}. Las tradicionales estructuras organizativas tienen su origen en la fragmentación de procesos naturales, producto de la división del trabajo, y la posterior agrupación de las tareas especializadas, resultantes en áreas funcionales o departamentos. Se caracterizan por una fuerte especialización individual y jerarquización por funciones, donde cada trabajador concentra su esfuerzo en la tarea que tiene asignada e intenta hacerla conforme a las instrucciones y especificaciones recibidas; pero con poca información e interés con relación al resultado final de su trabajo.

La estructura piramidal, válida para las organizaciones "en su tiempo", hoy por hoy, ya no compiten cuando se habla de calidad total en cada operación, en cada proceso; y se convierten en ineficientes por sus nichos de poder e inercia excesiva ante los cambios. La necesidad de contar con un sistema de control que posibilite la toma de decisiones basado no solo en el análisis económico - financiero [7], sino que logre una valoración integral de la gestión y sirva de herramienta en la ubicación de desviaciones en los diferentes procesos que la conforman [8]; la globalización de la economía que agudiza las presiones en cuanto a la competitividad [9]; la necesidad urgente de hacer empresas eficientes provocada por las exigencias crecientes de los clientes [10], conllevan a trabajar con las empresas e ir transformándolas desde adentro. Se impone la necesidad de cambios en la orientación estratégica de las organizaciones [11] en el perfeccionamiento de su sistema informativo [12] y la automatización de sus procesos [13].

Introducir la gestión por procesos es una tarea ardua y difícil. Por siglos organizaciones importantes y decisivas en la historia de la humanidad han presentado una estructura funcional. Más de cien años en el estudio y desarrollo de la Administración Científica y el reconocimiento de empresas exitosas, resultan razones de peso para el rechazo al cambio que se encuentra en la mayoría de las instituciones para la implementación de la gestión por procesos.

Lo anterior, a pesar de que los procesos han existido siempre, que resultan los encargados de realizar la transformación y la incorporación de valor y, por tanto, no resulta la misma historia para la "mejora de procesos" que desde el propio surgimiento de la ciencia del management, estuvo asociada a la mejora de las actividades y los procesos; pero nunca con una exigencia de alineación a la estrategia de las organizaciones como la exigida hoy en día.

La revisión de más de veinte conceptos de gestión por procesos entre los que se encuentran los planteados por Norma ISO (2008) [14], Bravo Carrasco (2009) [15], Maldonado (2011) [16], Salvador Oliván (2012) [17], Llanes Font (2014) [18], Aguilar Villanueva (2015) [19], Rey Peteiro (2015) [20] y Medina Nogueira (2016) [21], se pone de manifiesto que existe consenso en considerar a la gestión por procesos como la forma de gerencia de los procesos empresariales en sustitución de la gestión tradicional basada en las funciones y puede ser definida como: la forma de gestión de la organización basándose en los procesos en busca de lograr la alineación de los mismos con la estrategia, misión y objetivos, como un sistema interrelacionado destinado a incrementar la satisfacción del cliente, la aportación de valor y la capacidad de respuesta. Supone reordenar los flujos de trabajo de forma de reaccionar con más flexibilidad y rapidez a los cambios y en la búsqueda del ¿por qué? y ¿para quién? se hace el trabajo.

En consecuencia, son limitadas las organizaciones diseñadas y gestionadas por procesos, resulta más común que ambos tipos de forma de gestión coexistan y que este resultado sea logrado por la implementación de procedimientos de mejora de procesos en los que el logro de aspectos tales como: la definición de los procesos y para ellos, se reconozcan sus clientes, se formalicen sus actividades y se trabaje en el alineamiento con los objetivos de la organización sustentado en la mejora continua pueda ser considerado suficiente para 
comenzar. Sin dudas, esto implica una capacidad de transformar la cultura empresarial hacia el reconocimiento en cada actividad, de quiénes son sus clientes, sus necesidades y exigencias; así como el logro de la adecuada coordinación, sin feudos y fincas, que permitan la concreción de los objetivos estratégicos [22].

Pródigos resulta la cantidad de procedimientos y metodologías diseñados y encontrados en la literatura que abordan la "Mejora de Procesos". De su análisis, se puede concluir que poseen un grupo de etapas comunes, a saber: determinación de los procesos de la empresa, selección de los procesos para la mejora, representación de los procesos a ser mejorados, diagnóstico y determinación de las propuestas de mejora, implantación de las mejoras propuestas y seguimiento para la corrección de las desviaciones de las medidas implementadas [23].

Este análisis teórico y la experiencia práctica acumulada, conllevan a que resulta imposible abordar, de una sola vez, la mejora y transformación de toda la organización. Ciertamente, el objetivo será la mejora de todos los procesos de la empresa, pero es indudable que los recursos, fundamentalmente personal y tiempo, son finitos, lo que impone un orden para abordar la mejora.

En definitiva, el problema se reduce a fijar un grupo de criterios sobre la base de los que se decidirá el orden de los procesos a mejorar. Lógicamente, esta selección estará acompañada de herramientas que faciliten la determinación de este orden con rigor científico; pero, además, con la premisa de la búsqueda de una economía de tiempo al equipo encargado de realizar este proceso, entre los que se encuentran los técnicos y directivos más conocedores de la organización.

\section{ANÁLISIS TEÓRICO}

La investigación se sustenta en la revisión de más de 80 procedimientos para la mejora de procesos con el objetivo de encontrar invariantes, diferencias e incluso detectar posibles elementos no tratados en las propuestas anteriores y exigidos hoy, por el marco legal o los requerimientos de desarrollo de la sociedad. El análisis se basa en la aplicación del método estadístico de los Clúster y el software SPSS.
En la propuesta realizada aparecen otros métodos estadísticos, o específicos de este campo del conocimiento, que resultan herramientas de apoyo al procedimiento planteado, como resultan: métodos de expertos y criterios para su validación, el método Kendall, análisis multivariado, matriz modificada para analizar el cumplimiento de los objetivos estratégicos y repercusión en el cliente, entre otros.

Por último, el resultado obtenido es consecuencia de su enriquecimiento por la aplicación en la práctica social en más de 40 empresas de manufactura o servicios.

El trabajo tiene como objetivo proponer un procedimiento que permita crear un orden entre los procesos para su mejora, sustentado en la importancia que se le asocia a cada uno de ellos. De igual manera, se integran un conjunto de instrumentos estadísticos que facilitan el procesamiento y disminuyen la subjetividad de los resultados; a la vez que basada en una correcta representación de los procesos se garantice el enfoque al cliente, el alineamiento estratégico, la mejora continua y la implementación e integración de las normas ISO.

\section{RESULTADOS}

Es importante considerar que para la aplicación del procedimiento propuesto de "Mejora de Procesos" deberá cumplir con las premisas siguientes: existencia de compromiso por parte de la alta dirección, sustentado en un trabajo en equipo donde se involucre a especialistas con capacidad de aportar ideas y tomar decisiones.

De igual forma, el procedimiento propuesto tiene como objetivos:

1. Crear procesos que respondan a las estrategias y prioridades de la empresa y, por lo tanto, de sus clientes.

2. Conseguir que todos los miembros de la organización se concentren en los procesos adecuados.

3. Representar los procesos de la organización como base para lograr mejorar la eficacia, eficiencia y flexibilidad de los procesos para que el trabajo se realice de una forma más rápida, económica y centrada en aspectos esenciales. 
4. Crear una cultura que haga de la gestión de procesos una parte importante de los valores y principios de todos los miembros de la organización; un sistema de trabajo basado en la mejora continua.

La Figura 1 resumen la propuesta del procedimiento para la Gestión de procesos "DiANA", nombre que representa que la propuesta se centra en aquellos procesos de mayor impacto en el cumplimiento de los objetivos estratégicos y en las necesidades de los clientes ${ }^{4}$.

El presente artículo muestra en detalles el desarrollo de las tres primeras fases del procedimiento, que por demás son las que permiten una sistematización de la manera de actuar. Las herramientas y métodos a utilizar en las dos últimas fases están en un mayor grado condicionadas por las particularidades de los procesos que se estudien.

\section{Fase I: Organización}

\subsection{Planificación del proyecto}

Resulta necesario previo al trabajo establecer la planificación de las reuniones para el desarrollo del proyecto. Durante las actividades de trabajo grupal de esta etapa, se debe considerar como premisa que, es imprescindible:

$\checkmark$ La presencia del máximo líder de la organización.

$\checkmark$ El grupo debe estar integrado por personas con experiencia en la empresa, conocimientos técnicos de la actividad e iniciativas.

\subsection{Formación del equipo de trabajo}

Comprende la formación de un equipo de trabajo interdisciplinario compuesto por no más de siete u ocho personas, en función del tamaño de la empresa, en su mayoría miembros de la dirección de la empresa. Asimismo, deben poseer conocimientos en sistemas y herramientas de gestión, contar con la presencia de algún experto (interno o externo) acerca de la Gestión por Procesos y nombrar a un miembro del consejo de dirección como coordinador o facilitador del proyecto.

4 También intenta representar las iniciales de las personas que más han aportado al diseño y perfeccionamiento de este procedimiento.

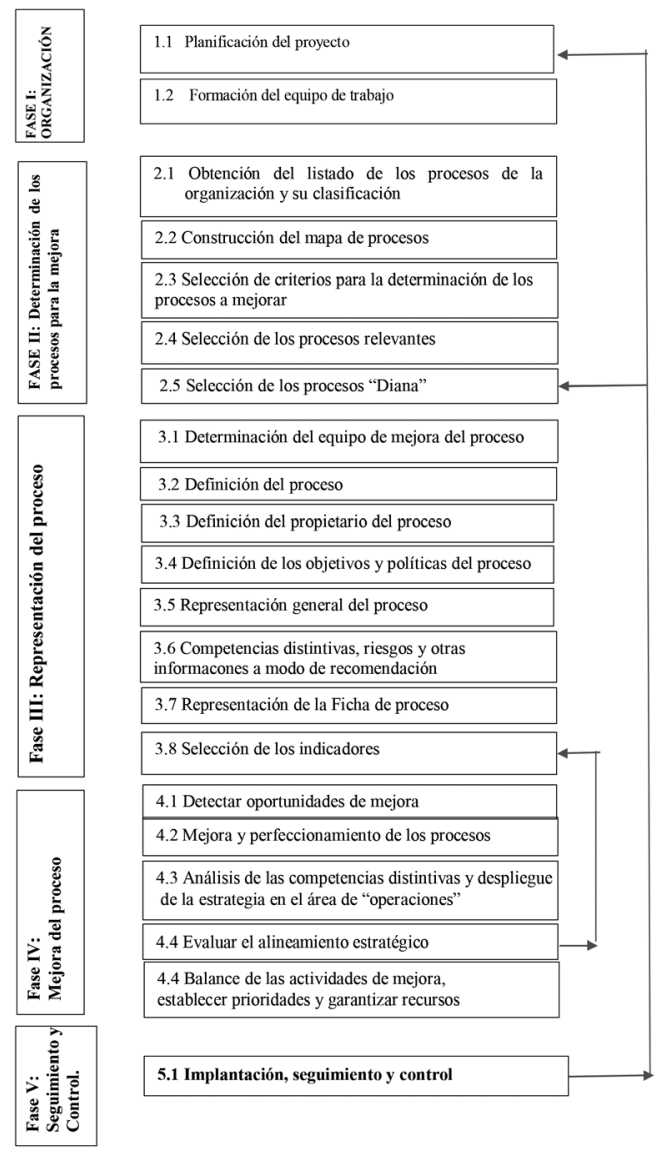

Figura 1. Procedimiento para la Gestión de procesos (DiANA). Fuente: resultado de la investigación.

\section{Fase II: Determinación de los procesos para la mejora}

\subsection{Obtención del listado de los procesos de la organización y su clasificación}

2.1.1. Obtención del listado de los procesos de la organización.

Antes de adentrarse en cualquier nueva iniciativa de gestión es esencial familiarizarse con los procesos empresariales internos propios de la empresa. Por tanto, en esta fase se recogerá, mediante una sesión de brainstorming, una lista de todos los procesos y actividades que se desarrollan en la empresa sobre la base de los postulados siguientes:

El nombre asignado a cada proceso debe ser sencillo y representativo de los conceptos 
y actividades incluidos en él. Asimismo, el proceso tiene que ser fácilmente comprendido por cualquier persona de la organización.

$\checkmark$ La totalidad de las actividades desarrolladas en la empresa deben estar incluidas en alguno de los procesos listados. En caso contrario deben tender a desaparecer.

$\checkmark$ Se recomienda que el número de procesos oscile entre 10 y 25 en función del tipo de empresa, pues la identificación de pocos o demasiados procesos incrementa la dificultad de su gestión posterior.

$\checkmark$ Se puede tomar como referencia otras listas afines al sector en el que se encuentra la empresa.

Para la sesión de trabajo del grupo para lograr el consenso acerca del listado de los procesos de la instalación se recomienda dividir al equipo de mejora en tres subgrupos. Previo a ello, o en la propia sesión se deben circular varios listados de procesos obtenidos de trabajos precedentes, en el sector o fuera de él; así como entidades internacionales de éxito, con el fin de servir de referencia. Todos los subgrupos no tienen por qué tener los mismos listados. Aclárese que estos listados son a manera de recomendación.

Cada subgrupo, desde su perspectiva, presenta la relación de los procesos conformados por ellos. Se recomienda el uso de una pancarta de manera de que se mantengan las tres propuestas al alcance de todos.

Posteriormente, se trabaja en la búsqueda del consenso para el listado de los procesos entre los equipos. Se plantea trabajar de lo más simple a lo complejo; hasta lograr el consenso.

Resulta imprescindible dejar definida la misión de cada uno de los procesos y proceder a su aprobación por el grupo. Se le solicita al director o al jefe del proyecto de mejora que realice una propuesta del responsable en elaborar en un párrafo la misión y límites del proceso. La aprobación es por el grupo. Este paso además de facilitar actividades posteriores (propietario del proceso, ficha, etc.) consolida el trabajo realizado hasta la fecha. Recuerde que, generalmente, existen intereses personales y una cultura funcional acentuada.

2.1.2. Clasificación de los procesos de la organización Primeramente, la clasificación de los procesos deberá estar en dependencia de la contribución que realizan a la actividad fundamental de la organización. La utilización posterior de esta clasificación en la elaboración del mapa de procesos es un tema coincidente por los autores que desarrollan esta ciencia [24-25], aunque en ocasiones con terminologías distintas. Se determina la utilización de: Procesos Estratégico, Procesos clave o misional, Procesos de apoyo o soporte.

Se procede entones a la clasificación de los procesos por cada grupo quienes publican su resultado en una pancarta, se buscan los puntos de contacto entre los tres equipos y se llega al resultado final por consenso.

\subsection{Construcción del mapa de procesos.}

El mapa de procesos es más que una representación gráfica de la secuencia e interacción de los procesos, resulta una aproximación que define la organización como un sistema de procesos interrelacionados que impulsa a la organización a poseer una visión más allá de sus límites geográficos y funcionales [17]; muestra cómo sus actividades están relacionadas con los clientes externos, proveedores y grupos de interés; da la oportunidad de mejorar la coordinación entre los elementos clave de la organización; un método para visualizar las actividades de una empresa, a todos los niveles, mediante los procesos ordenados por sus jerarquías y relaciones. Permite:

$\checkmark$ Elaborar un esquema general en el que se reflejen la totalidad de los procesos que se realizan en la empresa y las relaciones principales que se establecen entre ellos.

$\checkmark$ Responde a dos preguntas esenciales en el desarrollo de la mejora de los procesos, a saber: ¿Son todos los procesos que desarrolla la organización? ¿Existe alguna actividad que se realice en la organización y que no se encuentre reflejada en estos procesos?

$\checkmark$ Una excelente guía para el diseño de la estructura de la organización.

Ciertamente, aún resulta limitada la utilización del mapa de procesos como comparación de la estructura organizativa y del cumplimiento de la estrategia de la organización cuando debería ser una herramienta poderosa para este propósito. La estructura organizativa muestra o representa la manera en que se debe organizar la empresa, los procesos la forma en que se transforman las entradas en salida; ambos responden a la estrategia trazada. Por tanto, el mostrar en el 
mapa las principales relaciones entre los procesos en respuesta a la estrategia fijadas es de gran utilidad para el perfeccionamiento de la estructura organizativa. Para su creación se recomienda:

1. Cada miembro del equipo deberá plasmar las principales relaciones entre los procesos en una matriz "n x n", donde "n" es el número de procesos. Se les plantea a las personas vote sólo por cinco (5) relaciones en una escala de uno (1) a 10, donde 10 representa la relación más fuerte y 1 la más débil. Pueden repetirse los valores.

2. Realizar el consolidado de las tablas individuales por medio de la suma de los valores propuestos, respetar el criterio de considerar solo las cinco más relevantes relaciones. Esto no es un dogma, solo una guía de trabajo; pero el criterio recomendado está sustentado en el teorema de Euler y aplicado para lograr una representación en un solo nivel o plano.

3. Sesión de trabajo para obtener el consenso del equipo sobre la base del consolidado propuesto.

4. Construcción del mapa de Procesos. El mapa se crea con los tres niveles y las relaciones obtenidas El sentido de la relación, dado por fila y columna, se manifiesta en la saeta que une a los procesos donde la cola es la fila (origen) y la punta (columna) el destino. Existen procesos que poseen relaciones con fuerza en ambos sentidos y quedará reflejado con la existencia de saetas en las dos puntas de la flecha.

\subsection{Selección de criterios para la determinación de los procesos a mejorar.}

Se plantea plena concordancia con la matriz de objetivos estratégicos-repercusión en el cliente, para la determinación del orden de los procesos a ser mejorados. Esta matriz contiene los dos criterios más difundidos, dada la propia esencia de la gestión por procesos: alineación de los procesos con la estrategia (objetivos estratégicos) y orientación al cliente (repercusión en el cliente). Manifiesto de la forma siguiente:

Impacto del proceso (IP): Valoración de la incidencia en el cumplimiento de los objetivos estratégicos o metas de la organización.

Repercusión en el cliente (RP): Reflexión para cada proceso acerca de las incidencias que posee en la satisfacción de los clientes de la organización.
Otros criterios manejados en la literatura e incorporados a la selección de los procesos para la mejora son:

1. Posibilidad de éxito a corto plazo (ECP), basado en que se deben abordar primero aquellos procesos que más posibilidades tienen de alcanzar el éxito en el menor tiempo posible y, por tanto, ser más redituables [26].

2. Variabilidad (V) y Repetitividad (R), por ser las dos características esenciales que hacen importante el estudio de los procesos. Adicionalmente, las empresas que aplican de forma sistemática estas herramientas de mejora, con seguridad, llegarán al momento en que procesos de apoyo, por ejemplo, sean la causa principal de las insatisfacciones de los clientes, dado que los procesos claves o misionales ya mejorados reiteradamente, se encuentran ajustados y alineados. Evidente resulta que, en la medida que un proceso se repita más, será decisivo dedicarse a su mejoría. De ahí se puede definir: Variabilidad (V): cada vez que se repite el proceso hay ligeras variaciones en las distintas actividades realizadas que, a su vez, generan variabilidad en los resultados.

Repetitividad (R): los procesos se crean para producir un resultado e intentar repetir ese resultado una y otra vez. Esta característica permite trabajar sobre el proceso y mejorarlo: a más repeticiones más experiencia.

3. Valor agregado al producto final (VAPF), se utiliza para definir la cantidad que se incorpora al valor total de un bien o servicio en distintas etapas del proceso productivo, de distribución y de comercialización [27].

4. Peso Económico (PE), pues los recursos financieros constituyen un factor importante en el contexto de la limitación de recursos. Además, el costo del proceso debe ser estimado, no sólo por la carga de trabajo, sino también por la carga de recursos humanos y capital invertido [28].

5. Perfiles de Competencias (PC), se considera como proceso a ser mejorado aquel que posea el conjunto de conocimientos, habilidades, actitudes e intereses del personal de contacto en las empresas, cuya demostración en el desempeño de las funciones implica un determinado nivel de complejidad de los procesos mentales, con el consiguiente logro de los resultados esperados [29]. 
Como se aprecia, son varios los criterios que se pueden considerar en la selección de los procesos a ser mejorados. Además, resulta factible la incorporación de algún otro criterio que el equipo considere diferente a los mencionados. Se define como procesos "Diana", a aquellos en los que la entidad deberá centrar sus principales esfuerzos para la mejora [30].

\subsection{Selección de los procesos relevantes.}

Una vez establecido el listado de los procesos de la empresa por el equipo de mejora y presentados a la Alta Dirección, para su revisión y aprobación y como pre-selección a la obtención del orden de los procesos a ser mejorados (Diana), se debe aplicar el método del coeficiente de Kendall.

El algoritmo a seguir consiste en solicitar a cada experto su criterio acerca del ordenamiento según el grado de importancia de cada uno de los procesos. Para ello, el equipo deberá reflexionar acerca de los principales elementos que inciden en la selección de los procesos a mejorar (abordado en el paso anterior).

Este método permite reducir el listado de procesos (se les llama procesos relevantes) en función de su importancia y, a la vez, verificar la concordancia entre los juicios expresados por el grupo de expertos, con respecto a las evaluaciones que ofrecen para seleccionar los procesos relevantes. Con la aplicación de esta herramienta el listado se reduce a valores entre seis y ocho, lo que permite que el próximo análisis se realice con una mayor profundidad.

\subsection{Selección de los procesos "Diana"}

Para la selección de los procesos "Diana" se propone aplicar la matriz de objetivos estratégicos (IOE)/ repercusión en el cliente (RC)/ éxito a corto plazo (ECP); mostrada en la Tabla 1. Se incorporan, además, otros elementos o criterios que el equipo considere oportunos. A partir de ahí, se obtiene la puntuación total de cada proceso (TP).

Los criterios que formarán parte de la matriz serán aquellos que el equipo de mejora, con una valoración subjetiva, determinó como influyentes para la aplicación del método Kendall en la selección de los procesos relevantes.

Existe consenso en la literatura en cuanto a la importancia de las dos primeras variables, y la experiencia práctica ha demostrado que resulta viable considerar como factibles las cinco primeras. En cuanto a las restantes, se considera que están reflejadas en las primeras.

La solución de esta matriz puede ser abordada de dos formas distintas: la primera, bajo la consideración de que todos los criterios poseen la misma importancia y significación en cuanto al momento en que se aplica, o el sector al que pertenece la empresa; y la segunda, ofreciéndoles pesos relativos a cada uno de los criterios que denoten su importancia y significado que implica un procedimiento más complejo que el anterior, pero sin dudas, más preciso (análisis multivariado, Método Saaty).

La correlación establecida como variable de ponderación en la matriz confeccionada es: fuerte (10 puntos), media (5 puntos), y baja (1 punto). Una vez calculado el total de puntos para los procesos relevantes, el equipo selecciona los más significativos con referencia a los de máxima puntuación. Deberá realizarse el diseño o rediseño de todos los procesos

Tabla 1. Matriz modificada objetivos estratégicos - repercusión en el cliente.

\begin{tabular}{|c|c|c|c|c|c|c|c|c|c|c|}
\hline \multirow{2}{*}{ Procesos } & \multicolumn{3}{|c|}{$\begin{array}{l}\text { Objetivos } \\
\text { estratégicos } \\
\text { (IOE) }\end{array}$} & \multirow{2}{*}{\begin{tabular}{|c|}
$\begin{array}{c}\text { Total de } \\
\text { IOE }\end{array}$ \\
$\begin{array}{c}\text { Peso } \\
\text { Ponderado PP }\end{array}$ \\
\end{tabular}} & \multirow{2}{*}{$\begin{array}{c}\begin{array}{c}\text { Repercusión } \\
\text { en el cliente } \\
\text { (RC) }\end{array} \\
\text { PP }\end{array}$} & \multirow{2}{*}{$\begin{array}{c}\begin{array}{c}\text { Éxito a } \\
\text { corto plazo } \\
\text { (ECP) }\end{array} \\
\text { PP }\end{array}$} & \multirow{2}{*}{$\begin{array}{c}\text { Repetitividad } \\
\text { (R) }\end{array}$} & \multirow{2}{*}{$\begin{array}{c}\begin{array}{c}\text { Variabilidad } \\
\text { (V) }\end{array} \\
\text { PP }\end{array}$} & \multirow[t]{2}{*}{$\cdots$} & \multirow[t]{2}{*}{$\begin{array}{l}\text { Total de cada } \\
\text { proceso } \\
\text { (TP) }\end{array}$} \\
\hline & \begin{tabular}{l|l}
1 & 2
\end{tabular} & ... & $\mathbf{n}$ & & & & & & & \\
\hline 1 & & & & & & & & & & \\
\hline 2 & & & & & & & & & & \\
\hline$n$ & & & & & & & & & & \\
\hline
\end{tabular}

Fuente: en aproximación a (Amozarrain 1999), (Nogueira Rivera et al. 2004). 
relevantes (uno a uno) con inicio en los procesos Diana. La cantidad de procesos a ser mejorados de una vez depende de las particularidades de cada empresa y en especial de la fuerza de trabajo calificada que disponga para enfrentar esa labor.

\section{Fase III: Representación del proceso}

Realizar la representación del proceso presupone la ejecución de un conjunto de pasos que logran su resultado o materialización final en la Ficha de proceso y de indicadores. A continuación, se expone un esquema que sintetiza las actividades a realizar (Ver Figura 2).

\subsection{Determinación del equipo de mejora del proceso} El equipo de trabajo deberá estar constituido por grupo de personas propuestas por el jefe del equipo de mejora, en cantidad entre seis y ocho en función de la complejidad del proceso y tipo de organización, con conocimiento del proceso y posibilidad de aportar ideas y criterios, liderados por el propietario del proceso. En lo posible es importante contar con la presencia de personas que resulten suministradores o clientes del proceso que se analiza.

\subsection{Definición del proceso}

El equipo debe definir el alcance del proceso empresarial objeto de estudio y su relación con otros procesos que la organización utiliza para planificar, ejecutar, revisar y adaptar su comportamiento (ciclos $\mathrm{P}, \mathrm{D}, \mathrm{C}, \mathrm{A})$, de manera que todos estén de acuerdo con el trabajo que deben realizar. A ello contribuye la confección de una ficha, denominada ficha del proceso, que incluye los elementos del proceso que se mencionan a continuación: nombre, responsable, finalidad, objetivos, clientes, proveedores, otros grupos de interés, procesos relacionados y contenido.

En este punto se deberá resumir: misión y objetivos, clasificación y caracterización del sistema límites, entradas y salidas, competencia distintiva. En procesos de servicio considerar criterios como nivel de contacto,

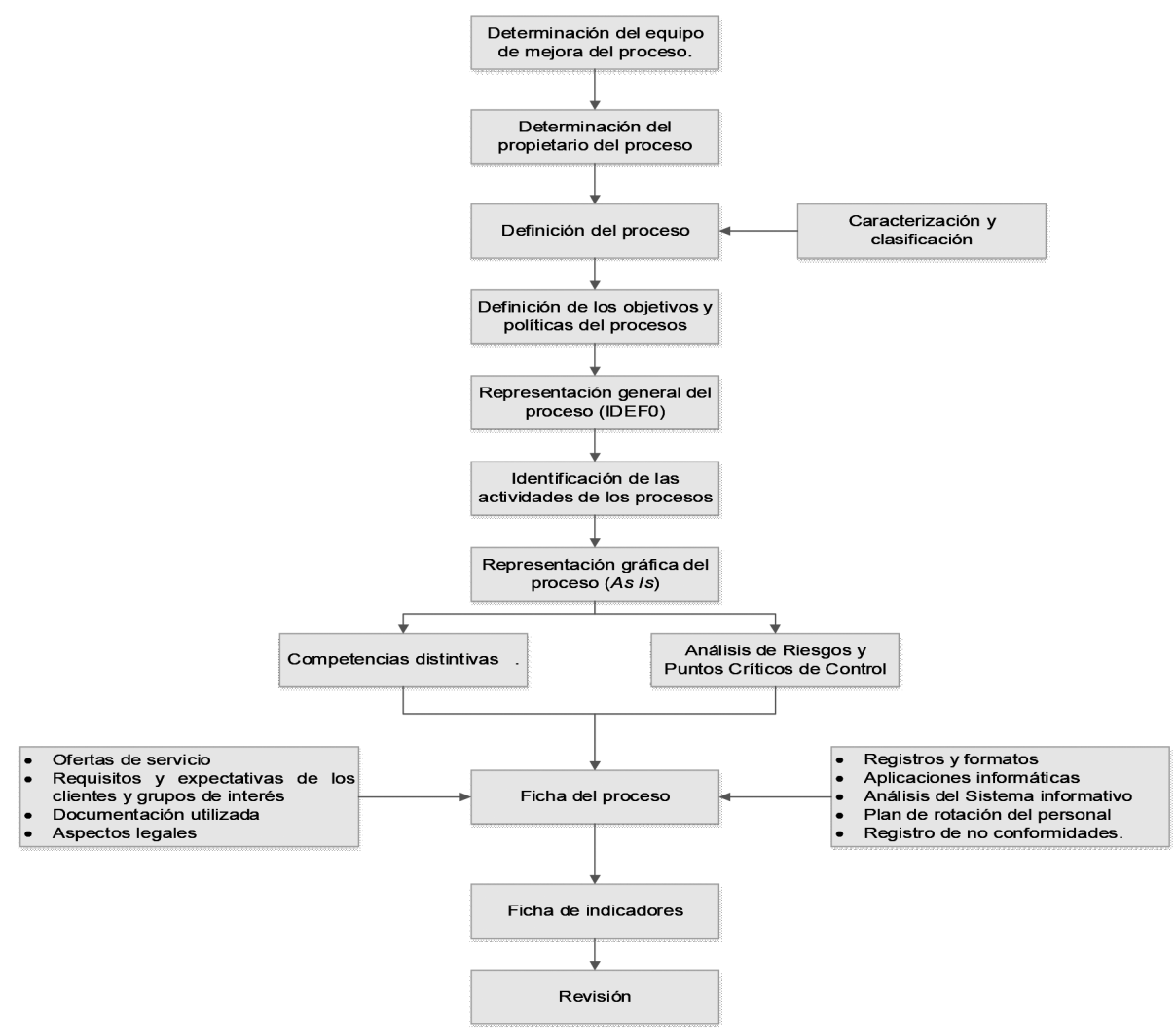

Figura 2. Procedimiento propuesto para la representación de procesos y su concreción en las Fichas del proceso e indicadores. 
relación cliente/mercado (case mix), grado de interacción y adaptación junto al grado de intensidad de la mano de obra Schmener (1986) ápud [31]. Estas clasificaciones son necesarias por su influencia en las decisiones de operaciones, y en el diseño y mejora de procesos.

Para establecer los objetivos básicos del proceso se debe rescatar la información contenida en la matriz utilizada para la selección de los procesos Diana para constatar que:

Esté alineada con los objetivos estratégicos. El equipo debe lograr el despliegue de los objetivos estratégicos a través del proceso.

$\checkmark \quad$ Las necesidades de los clientes. Se analiza la repercusión del cumplimiento de las necesidades de los clientes del proceso, a través de: entrevistas, encuestas u observaciones directas sobre sus expectativas [32].

\subsection{Definición del propietario del proceso}

El propietario del proceso es nombrado por la alta dirección de la organización y puede o no ocupar responsabilidades funcionales. Debe poseer un amplio dominio del proceso $y$, sobre todo, capacidad para analizar con enfoque sistémico y en función del cumplimiento de los objetivos estratégicos. En muchas ocasiones una excelente medida para un área de la organización perjudica el resultado general del proceso y en estas situaciones es decisiva la existencia y participación adecuada del propietario del proceso.

\subsection{Definición de los objetivos y políticas del proceso} La definición de los objetivos y políticas del proceso resulta una exigencia de las normas ISO. Se recomienda que se analice su definición desde los enfoques estratégicos, de calidad y ambiental.

\subsection{Representación general del proceso}

Se representa gráficamente cada proceso en aproximación a un mapa IDEF0 $^{5}$ (Figura 3). Se representan los elementos relevantes para su comprensión a nivel general (entrada, mecanismos, controles y salidas). En este paso se facilita además la búsqueda de la información asociada a: suministradores, se destacan los recursos necesarios para el proceso; infraestructura (con información acerca de la capacidad del proceso y los cuellos de botella); grupos de interés asociados al proceso; clientes/destinatarios (conocidas y definidas las ofertas de servicio y los requisitos o expectativas del cliente y de otros grupos de interés); el análisis de los residuos, el manejo de las sustancias tóxicas o peligrosas [33].

Es importante además determinar las ofertas de servicio y referirse a aquellas particularidades que debe poseer el proceso para su ejecución exitosa, por ejemplo: confiabilidad, celeridad, trazabilidad del estado del trámite.

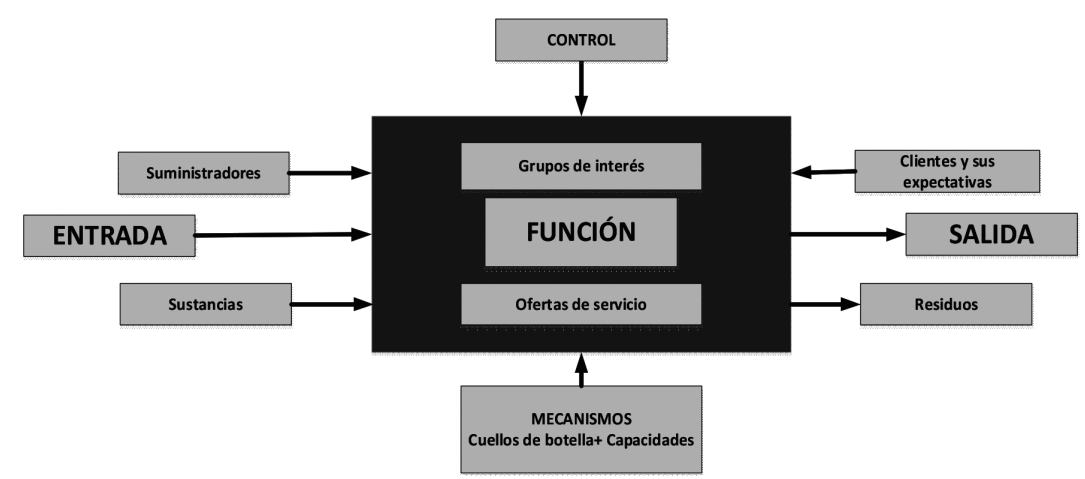

Figura 3. Representación general del proceso en aproximación a un IDEFo, fertilzados con otras exigencias normativas.

5 El mapa IDEF0 es la descripción gráfica, de un sistema o contenido, que se desarrolla con un propósito concreto y un punto de vista determinado. El conjunto de uno o más diagramas IDEF0 describe las funciones del área de un sistema o sujeto con gráficos, textos y glosarios. 
3.5.1. Identificación de las actividades de los procesos Los procesos de una organización pueden ser divididos en subprocesos y actividades. La identificación detallada de cada actividad es importante para una correcta definición de los procesos y lograr una representación gráfica efectiva. Se puede realizar de dos maneras:

1. El líder del equipo prepara un diagrama preliminar de acuerdo a su experiencia y conocimiento personal del proceso, que puede ser confirmado por otras personas que conozcan el proceso y no formen parte del equipo. Finalmente, el equipo revisa y actualiza el diagrama en una sesión de grupo.

2. El otro método y más utilizado, es aquel en el que el líder del equipo u otra persona capacitada organiza una sesión de brainstorming para desarrollar la descripción desde cero y en base a los conocimientos y experiencias de los miembros del equipo.

Surgen preguntas que ayudarían a esclarecer la identificación y determinación de las actividades que conforman el proceso: ¿Cuáles suceden siempre?; ¿Cuáles suceden a veces?

\subsubsection{Representación gráfica de los procesos}

Los diagramas de procesos, son los métodos utilizados para representar los procesos con el fin de conocerlos y por consiguiente mejorarlos. Dos formas muy difundidas en la actualidad y recomendadas para su uso de forma conjunta resultan: Diagramas IDEFo y el As Is (Tal como es).

No importa la técnica que se adopte, la representación de procesos por medio de mapas, conlleva varias fases; y diversos autores aportan procedimientos para su ejecución y se apoyan en: entrevistas con ejecutantes del proceso; mostrar las actividades que realmente ocurren; documentar sobre errores o fallas que se cometen en el proceso; determinar riesgos y puntos críticos de control, inventario de momentos de verdad; protocolos (prácticas clínicas, variabilidad), normas; tiempo del ciclo; costos.

La mayoría de los procedimientos aboga por un primer paso que comprenda la delimitación y definición del proceso (bien podría apoyarse en aspectos como caracterización y clasificación de sistemas productivos; para, posteriormente, identificar las actividades o procesos (en dependencia del nivel de detalle) y su secuencia hasta producir el resultado final.

Para completar un Diagrama de Flujo de Procesos se deben definir los símbolos a utilizar (se recomienda los utilizados por el diagrama Visio). Con cada símbolo se coloca la descripción de la actividad.

Para simplificar su elaboración se recomienda utilizar una técnica basada en una estructura de frases sencillas para etiquetar cada una de las etapas del diagrama. Esta técnica se conoce como Paradigma: Recurso - Acción - Objeto [8]. Consiste en definir el sujeto de la acción, o sea, el que la realiza (Recurso); la acción a realizar representada por un verbo (Acción); y el objeto de la acción (Objeto). Por ejemplo: Contabilidad (Recurso) revisa (Acción) la orden del cliente (Objeto).

\subsection{Competencias distintivas, riesgos y otras infor- maciones a modo de recomendación}

3.6.1. Determinación de las competencias distintivas Una competencia distintiva debe traducirse en resultados que sean valiosos para el cliente y que satisfagan la estrategia de la organización. Exige que operaciones se concentre en la tarea que debe dominar, ya que es frecuente que no se puedan dominar todas las dimensiones empresariales a la vez.

A pesar de ser un tema abordado por clásicos de la Gestión de Operaciones aún su consideración en las estrategias organizacionales y en la propia mejora de los procesos resulta limitada [21].

\subsubsection{Identificación de los riesgos}

La identificación de los riesgos por procesos es el paso inicial para la evaluación de posibles vulnerabilidades. La reducción o eliminación de los riesgos, principalmente los de alta probabilidad de ocurrencia y de un mayor impacto, garantiza una seguridad razonable en la organización. Insertar dentro de la ficha de proceso los riesgos empresariales, es un punto importante para lograr una verdadera integración en la función de control.

El poseer registro de los accidentes, incidencia y riesgos o eventos casuales permiten su gestión y prevención. Se recomienda un registro que contemple: hecho perjudicial, fecha, descripción, consecuencias y causas que se le pueden asociar. 
3.6.3. Otras informaciones a modo de recomendación La elaboración de la Ficha de procesos o los manuales poseen la utilidad de garantizar el desarrollo del trabajo de una forma correcta y sistemática; pero sobre todo la gestión del conocimiento. Estas herramientas permiten perpetuar las buenas prácticas a pesar de la salida de la organización del personal de más experiencia y que tradicionalmente "ha sabido hacer" las cosas. En contraposición su confección es difícil, no siempre bien vista por el personal de trabajo y más difícil aún su mantenimiento con actualidad.

Por tanto, a continuación, se reflejan un conjunto de aspectos tendientes a que las fichas de procesos brinden toda la mayor información posible. Se recomienda que su incorporación a la Ficha sea paulatina y como parte del propio proceso de mejora continua. Estas son: ofertas de servicios, requisitos (expectativas) del cliente y otras partes interesadas, documentación utilizada, aspectos legales (normas, leyes, procedimientos establecidos y que deben ser cumplidos en el puesto de trabajo), registros y formatos (documentos creados por la propia oficina para facilitar la labor), aplicaciones informáticas, sistema informativo, plan de rotación del personal y propuesta de un registro de incidencias de las no conformidades.

\subsection{Representación de los resultados en la Ficha de proceso}

Para culminar la definición de los procesos se realiza una ficha que incluya las características relevantes definidas en el diagrama, así como la gestión del proceso. La información a incluir dentro de una ficha de proceso es diversa y debe definirse por la propia organización. Las principales cualidades que fueron establecidas en pasos anteriores y otras necesarias para el buen desempeño de una gestión por procesos se muestran en el Cuadro 1.

Cuadro 1. Ficha de procesos propuesta para una efectiva gestión por procesos en las organizaciones.

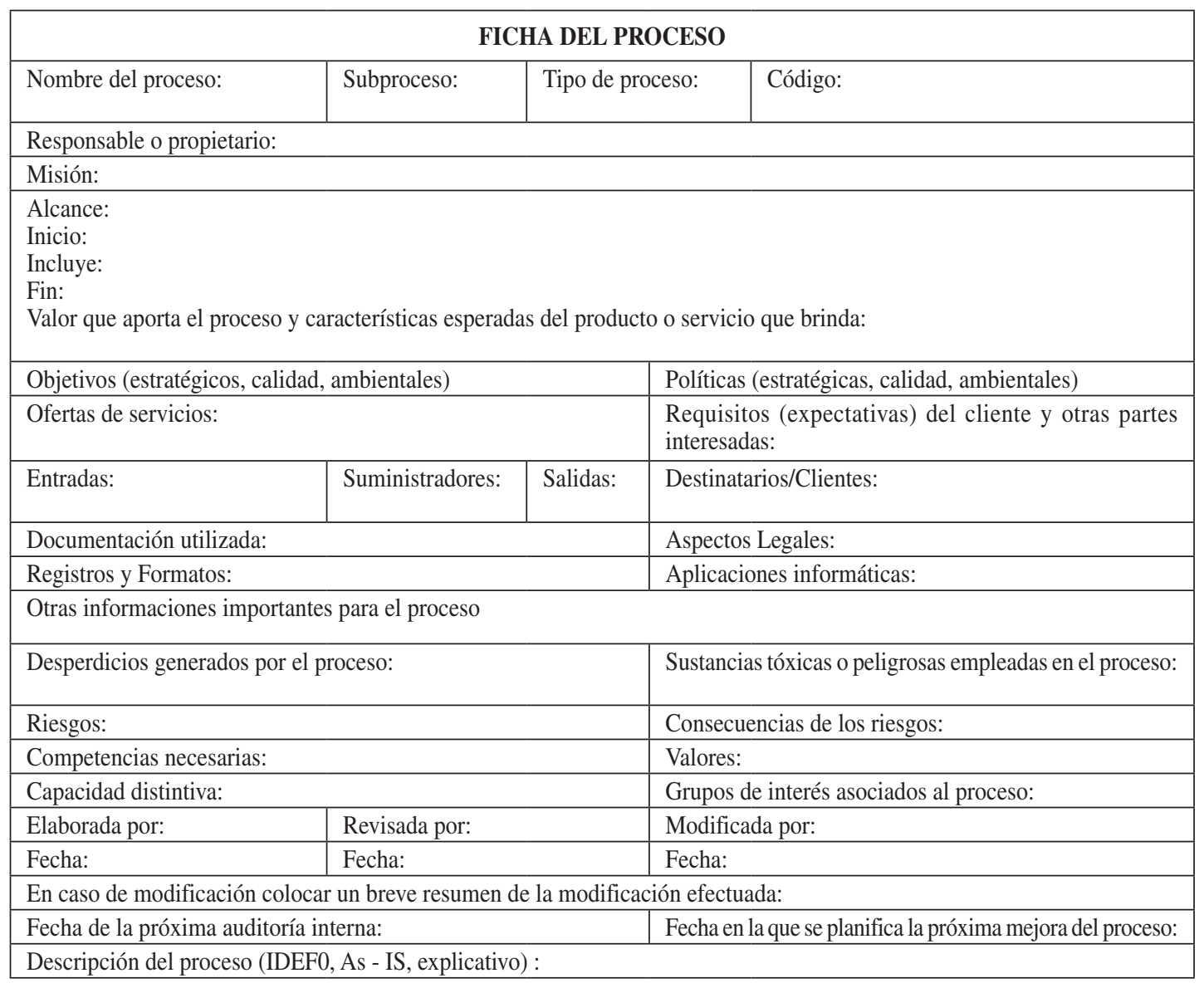

Fuente: (Medina León et al. 2014). 


\subsection{Selección de los indicadores}

El indicador es el instrumento de medición para valorar el desempeño del proceso. Se define el listado de los indicadores, de eficiencia y eficacia, para la evaluación de los procesos y del desempeño empresarial, recomendablemente a través del Cuadro de Mando Integral (CMI). Para una formalización de los indicadores se deben definir, al menos, las características siguientes: nombre, objetivo del indicador, forma de cálculo, unidad de medida, punto de lectura, periodicidad y niveles de referencia.

Todos estos valores son representados en una ficha de indicador como complemento de la ficha de proceso que se muestra en Cuadro 2.

\section{Fase IV: Mejora del proceso}

La fase de mejora debe ser analizada con un enfoque en sistema [34]. En ocasiones, lo que representa una mejora para un área funcional no lo resulta para el sistema en su conjunto, la estrategia o a las necesidades de los clientes [35]. Esta mejora en los finales del siglo pasado se centraba en la eliminación de las actividades que no aportan valor añadido, posteriormente con el surgimiento de las normas ISO tomaron relevancia los enfoques centrados en la calidad [36]. Hoy por su parte, sin descuidar los anteriores y otras formas de mejora se centra la atención en la automatización de los procesos y en el logro del alineamiento estratégico.

Si bien resulta tradicional comenzar por buscar eliminar aquellas actividades que no aportan valor añadido, analizar las más costosas o duraderas, hacer coincidir los equipos fundamentales con los limitantes o buscar las necesidades de los clientes para convertirlas en exigencias del proceso, no existe un orden establecido para seleccionar las herramientas a aplicar, sin dudas, depende del sistema que se estudie y sus problemas.

La mejora resulta un paso decisivo dado que no solo contribuye a la búsqueda de la eficiencia del sistema, sino que también a implementar los sistemas de gestión o todas aquellas tendencias modernas asociadas a la gestión por procesos, a saber: normas ISO [37], integración de sistemas, puntos críticos de control, análisis de riesgos [38], benchmarking, modelo EFQM, CMI, etcétera; así como, para el logro de la efectividad con el uso de herramientas como la innovación, la gestión del conocimiento, la gestión por competencias, la implantación de la responsabilidad social, entre otras. Algunos pasos generales recomendados resultan:

$\checkmark$ Detectar oportunidades de mejora.

$\checkmark$ Seleccionar las herramientas o vías para el logro de la mejora.

$\checkmark$ Análisis de las competencias distintivas u otras formas de potenciar la actividad de "operaciones" en los procesos (fundamentalmente para los claves).

$\checkmark$ Evaluar el grado de alineamiento estratégico y tomar acciones para su mejora.

$\checkmark$ Análisis de las competencias distintivas u otras formas de potenciar el despliegue de la estrategia en el área de "operaciones" en los procesos (fundamentalmente para los claves).

Cuadro 2. Propuesta de ficha de indicador como complemento a la ficha de procesos.

\begin{tabular}{|c|c|c|c|}
\hline \multicolumn{4}{|c|}{ FICHA DE INDICADOR } \\
\hline \multicolumn{3}{|l|}{ Indicador: } & Eficiencia: \\
\hline \multicolumn{3}{|l|}{ Utilizado en la Gestión para: } & Eficacia: \\
\hline \multicolumn{3}{|c|}{ Expresión de cálculo y/o descripción de la forma de obtenerlo: } & Unidad de medida: \\
\hline Donde se obtiene: & \multicolumn{3}{|c|}{ Cuando se obtiene (y la frecuencia en caso de ser necesario): } \\
\hline \multicolumn{4}{|l|}{ Fuente de la información: } \\
\hline \multicolumn{4}{|l|}{ Resultado planificado: } \\
\hline $\begin{array}{l}\text { Resultado de la competencia u otras empresas } \\
\text { del sector: }\end{array}$ & \multicolumn{2}{|c|}{ Resultados de la empresa líder: } & Gráfico de tendencias: \\
\hline \multicolumn{4}{|l|}{ Registros y Formatos: } \\
\hline Elaborado por: & \multicolumn{2}{|c|}{ Revisado por: } & Modificado por: \\
\hline Fecha: & Fecha: & Fecha: & \\
\hline
\end{tabular}

Fuente (Medina León et al. 2014). 


\section{Fase V: Seguimiento y Control}

\subsection{Implantación, seguimiento y control}

Como apunta Amozarrain (1999) ápud [20]: "La fase de implantación puede prolongarse en el tiempo, por lo que es necesario desarrollar un plan concreto con la definición de responsables y plazos para cada uno de los hitos".

Antes de implantar el nuevo proceso es necesario reflexionar acerca de las posibles resistencias al cambio y las posibles contramedidas a adoptar de entre las que se pueden citar las siguientes:

\section{$\checkmark$ Comunicar y hacer partícipes a las personas que se verán implicadas en la puesta en práctica del nuevo proceso. \\ $\checkmark$ Dar la formación y adiestramiento necesarios. \\ $\checkmark$ Escoger el momento adecuado. \\ $\checkmark$ Desarrollar una implantación progresiva, que procure iniciar ésta con las personas más receptivas y con las de más prestigio entre sus compañeros.}

\section{CONCLUSIONES}

Se presenta un procedimiento elaborado para el análisis y mejora de los procesos como resultado del estudio de más de 80 referencias anteriores y su propio enriquecimiento producto de la aplicación en más de 40 empresas de manufactura o servicio. Las principales bondades de la propuesta se centran en su capacidad de actuar primero sobre aquellos procesos que inciden de una manera decisiva en el cumplimento de los objetivos estratégicos y posean un mayor impacto en los clientes bajo un proceso de mejora continua.

La propuesta incorpora un conjunto de herramientas que le aportan valor científico, a la vez que procura para los miembros del equipo de mejora (principales líderes de la organización) un manejo eficiente del tiempo y una participación activa con el consecuente aprendizaje y compromiso con las soluciones brindadas.

La propuesta dedica un esfuerzo notable a la manera de lograr una efectiva representación del proceso como base para la mejora, la incorporación de los diferentes sistemas de gestión o su integración. Garantiza por demás, la gestión del conocimiento al formalizar en detalle la manera de hacer, normativas legales, instrumentos, indicadores y otros elementos importantes de los procesos.

\section{REFERENCIAS}

[1] M. Cousins. "What's in a process map?". 2000. Fecha de consulta: Marzo, 2012. Disponible en: http://www.Iqa.org.publication/c4-1-80. shtml.

[2] J. Zaratiegui. "La gestión por procesos: su papel e importancia en la empresa". Revista Economía Industrial. Vol. VI N 330 , pp. 81-88. 1999. ISSN: 0422-2784.

[3] A. Hernández Nariño, D. Nogueira Rivera, A. Medina León y M. Marqués León. "Inserción de la gestión por procesos en instituciones hospitalarias: concepción metodológica y práctica”. Revista de Administração (São Paulo). Vol. 48 No 4, pp. 739-756. 2013. ISSN: 0080-2107. http://www.scielo.br/ scielo.php?script=sci_arttext\&pid=S008021072013000400009\&lang=pt.

[4] M.D. Arango Serna, L.F. Campuzano Zapata y J.A. Zapata Cortes. "Mejoramiento de procesos de manufactura utilizando Kanban". Revista Ingenierías Universidad de Medellín. Vol. 14 No 27, pp. 221-233. 2015. ISSN: 1692-3324. http://www.scielo.org.co/ scielo.php?script=sci_arttext\&pid=S169233242015000200014\&lang=pt.

[5] J.C. Flores Santander, E. Pérez Adán, A. Medina León, D. Nogueira Rivera y M. Oviedo Rodríguez. "Enfoque de procesos para la reducción de paros de máquinas mediante mantenimiento centrado en confiabilidad. Impacto en la economía como rama de las ciencias sociales". Revista Magazine de las Ciencias. Vol. $2 \mathrm{~N}^{\circ} 4$ pp. 31-42. 2017. ISSN: 2528-8091.

[6] H.F. Castro Silva y F. Rodríguez. "Incidencia de la certificación de la norma ISO 9001 en los resultados empresariales. Un caso colombiano". Revista Entre Ciencia e Ingeniería. Vol. $11 \mathrm{~N}^{\circ} 22$, pp. 18-25. 2017. ISBN: 1909-8367. http://www.scielo.org.co/ scielo.php?script=sci_arttext\&pid=S190983672017000200018\&lang=pt.

[7] R.S. Kaplan and D.P. Norton. "The execution premium: integrando la estrategia y las operaciones para lograr ventajas competitivas". 
Editorial Deusto. Barcelona, España. pp. 392. 2008. ISBN: 978-84-234-268I-2.

[8] W. Trischler. "Mejora del valor añadido en los procesos". Ediciones Gestión 2000 S.A. Barcelona, España. pp. 155. 1998. ISBN: 848-082-22-20.

[9] I. Elia Damian y M.F. Suárez Barraza. "Innovación de procesos en la gestión turística: Una revisión de la literatura". Revista Intangible Capital. Vol. $11 \mathrm{~N}^{\mathrm{o}} 2$, pp. 147-165. 2015. ISSN: 1697-9818

[10] I. Cantón Mayo. "Introducción a los Procesos de Calidad". Revista Iberoamericana sobre Calidad, Eficacia y Cambio en Educación (REICE). Vol. $8 \mathrm{~N}^{\circ}$ 5, pp. 45-53. 2016. ISSN: 1696-4713.

[11] A. Alonso Becerra, E. Michelena Fernández y D. Alfonso Robaina. "Dirección por procesos en la Universidad". Revista Ingeniería Industrial. Vol. $34 \mathrm{~N}^{\mathrm{o}}$ 1, pp. 87-95. 2013. ISSN: 1815-5936. http://scielo.sld.cu/ scielo.php?script=sci_arttext\&pid=S181559362013000100009\&lang=pt.

[12] L.B. Alpízar Caballero, H. Trutié Rodríguez, C.A. Sarría Pérez y A.M. Pérez Sánchez. "Sistema de Información para la Gestión de Ciencia, Tecnología e Innovación en las Facultades de Ciencias Médicas". Revista Cubana de Medicina Militar. Vol. $44 \mathrm{~N}^{\circ} 1$, pp. 96-104. 2015. ISSN: 0138-6557. http://scielo.sld.cu/scielo. php?script $=$ sci_arttext\&pid $=$ S013865572015000100011\&lang=pt.

[13] M. Arias y E. Rojas. "Guía para gestionar procesos de negocio a través de minería de procesos". Revista InterSedes. Vol. 17 $\mathrm{N}^{\mathrm{o}} 36$, pp. 1-28. 2016. ISSN: 22152458. http://www.scielo.sa.cr/scielo. php? script $=$ sci_arttext $\&$ pid $=$ S 2215 24582016000200001\&lang=pt.

[14] N. ISO 9001 2008. Norma Internacional ISO 9001: 2008, Secretaria Central de ISO, 2008.

[15] J. Bravo Carrasco. "Gestión avanzada de Procesos". Editorial Evolución S. A. Santiago de Chile, Chile. pp. 185. 2009. ISBN: 978-956-7604-16-6.

[16] J.A. Maldonado. "Gestión de Procesos". Editorial Eumed.net. URL: www.eumed. net/libros- -gratis/2011e/1084/indice.htm. pp. 245. 2011.
[17] J.A. Salvador Oliván y M.J. Fernández Ruiz. "Mapa de procesos de un sistema de gestión de accesibilidad en un Servicio Web de la administración pública: El ayuntamiento de Zaragoza". Revista El profesional de la información. Vol. $21 \mathrm{~N}^{\mathrm{o}}$ 3, pp. 6-8. 2012. ISSN: 1386-6710.

[18] M. Llanes Font, C.L. Isaac Godínez, M. Moreno Pino y G. García Vida. "De la gestión por procesos a la gestión integrada por procesos". Revista Ingeniería Industrial. Vol. XXXV No 3, pp. 255 -264. 2014. ISSN: 1815-5936.

[19] L.F. Aguilar Villanueva. "Gobernanza y gestión pública". Fondo de cultura Económica. D.F., México. pp. 160. 2015. ISBN: 6-071-633-648.

[20] D. Rey Peteiro. "Todo sobre la Gestión por Procesos (Parte I)”. [en línea], 2012, pp. 45-52 [consulta: Disponible en: <URL: www.w3.org/ TR/xhtml1/DTD/xhtml1-strict.dtd>

[21] D. Medina Nogueira, D. Nogueira Rivera, A. Medina León y J. Suárez Hernández. "Procedimiento para el diagnóstico de la gestión del conocimiento". Revista Retos de la Dirección. Vol. 10 Nº 2, pp. 168-192. 2016. ISSN: 2306-9155.

[22] O. López Batistas, A. Medina León, D. Nogueira Rivera, A. Hernández Nariño y J.C. Zulueta Cuesta. "Acercamiento a la responsabilidad social universitaria desde un enfoque de procesos y basada en la gestión del conocimiento". Revista Anuario de la UISEK. Vol. $4 \mathrm{~N}^{\mathrm{o}}$ 12, pp. 61-68. 2012.

[23] A. Medina León, D. Nogueira Rivera, A. Hernández Nariño y Y. Díaz Navarro. "Consideraciones y criterios para la selección de procesos para la mejora: Procesos Diana". Revista Ingeniería Industrial. Vol. XXXIII $\mathrm{N}^{\circ}$ 3, pp. 272-281. 2012. ISSN: 1815-5936.

[24] E. Negrín Sosa. "El mejoramiento de la Administración de Operaciones en Empresas de Servicios Hoteleros". Tesis para optar al grado de doctor. Universidad de Matanzas. Matanzas, Cuba. 2003.

[25] G. Ponjuán Dante. "Principios y métodos para el mejoramiento organizacional". Editorial Félix Varela. Ciudad de La Habana, Cuba. pp. 192. 2006. ISBN: 959-07-0193-0.

[26] D. Nogueira Rivera, A. Medina León y C. Nogueira Rivera. "Fundamentos para el 
control de la gestión empresarial". Editorial Pueblo y Educación. Ciudad de La Habana, Cuba. 2004. ISBN: 959-13-1192-3.

[27] N. Machado Rodríguez. "El Control de Gestión en instituciones bancarias". Tesis para optar al grado de doctor. Universidad de la Habana. La Habana, Cuba. 2003.

[28] J.P. Claveranne and C. Pascal. "Repenser les processus a l'hopital. Une methode au service de la performance". Editorial Médica Editions. Paris, Francia. pp. 215. 2004. ISBN: 2-914357-03-06.

[29] C. Parra Ferié. "Modelo y procedimientos para la gestión con óptica de servucción de los servicios técnicos automotrices como elemento del sistema turístico cubano". Tesis para optar al grado de doctor. Instituto Superior Politécnico José Antonio Echeverría. La Habana, Cuba. 2005.

[30] A. Solé Cabanes. "Gestión por Procesos". 2008. Fecha de consulta: 20 de septiembre de 2017. Disponible en: http://www.budok. es/tienda/libros-tags/iso9001

[31] R.G. Schroeder, S. Meyer Goldstein y M.J. Rungtusanatham. "Administración de operaciones: conceptos y casos contemporáneos". McGraw-Hill. Interamericana Editores, S.A. de C. V. 5taEdición. D.F, México. pp. 542. 2011. ISBN: 978-0-07-340338-0.

[32] J.W. Cruz Riveros y O.J. Gómez. "Percepción del enfermero en procesos estratégicos de programas de protección específica y detección temprana en IPS de Bogotá". Revista Avances en Enfermería. Vol. 31 No 1, pp. 11-21. 2013. ISSN: 0121-4500. http://www.scielo.org.co/ scielo.php?script=sci_arttext\&pid=S012145002013000100002\&lang=pt.
[33] A. Medina León. "La documentación de procesos y su formalización, herramienta para la integración de los sistemas de gestión y la sustentabilidad", en Organizaciones Sustentables. Cuerpo Académico Procedimientos Contables y Administrativos en el ámbito de la Organización, de Teodora González Rodríguez y Mireia Valverde Universidad Veracruzana. Primera Edición. Veracruz, México. pp. 127143. 2014. ISBN: 978-607-9248-63-5.

[34] R.R. Alcalde Miller y J.I. Chavez Galvez. "Mejora en el proceso de conciliación y liquidación de medios de pago en Supermercados Peruanos SA”. 2015.

[35] H. Ricardo Cabrera, A. Medina León, J. Abab Puente, D. Nogueira Rivera, O. Sánchez Díaz y Q. Nuñez Chaviano. "Procedimiento para la Identificación y Evaluación de las oportunidades de mejora: medición de la factibilidad e impacto". Revista Ingeniería Industrial. Vol. 37 $\mathrm{N}^{\mathrm{o}}$ 1, 2016. ISSN: 0258-5960.

[36] H. Ricardo Cabrera, A. Medina León, C.M. Pérez Hernández, Q. Núñez Chaviano y M. Curbelo Hernández. "Mejora de la disponibilidad en procesos a partir de la confiabilidad". Revista Anuario Facultad de Ciencias Económicas y Empresariales. Vol. 7 $\mathrm{N}^{\circ}$ 1, pp. 18-33. 2015. ISSN: 2218-3639.

[37] M.M. Bernal Zipa. "Gestión por procesos y mejora continua, puntos clave para la satisfacción del cliente". Universidad Militar Nueva Granada. 2015.

[38] P. Bazán, J.M. Garro, R. Giandini y J. Diaz. "Mejora de la monitorización y ejecución de procesos de negocio con integración y socialización". Computing Conference (CLEI), 2015 Latin American. 